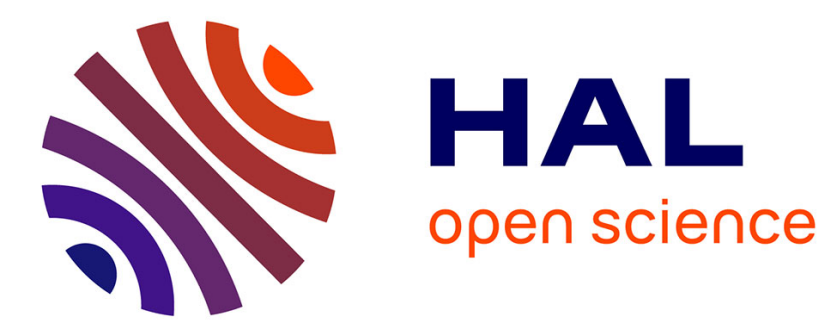

\title{
École, jeunes de milieux populaires et groupes de pairs
}

Mathias Millet, Daniel Thin

\section{To cite this version:}

Mathias Millet, Daniel Thin. École, jeunes de milieux populaires et groupes de pairs. Mucchielli, Laurent. Les bandes de jeunes. Des " blousons noirs " à nos jours, La Découverte, 2007, Recherches. halshs-00971501

\section{HAL Id: halshs-00971501 https://shs.hal.science/halshs-00971501}

Submitted on 24 Dec 2021

HAL is a multi-disciplinary open access archive for the deposit and dissemination of scientific research documents, whether they are published or not. The documents may come from teaching and research institutions in France or abroad, or from public or private research centers.
L'archive ouverte pluridisciplinaire HAL, est destinée au dépôt et à la diffusion de documents scientifiques de niveau recherche, publiés ou non, émanant des établissements d'enseignement et de recherche français ou étrangers, des laboratoires publics ou privés. 


\section{7. ÉCOLE, JEUNES DE MILIEUX POPULAIRES ET GROUPES DE PAIRS}

Mathias Millet, Daniel Thin

in Marwan Mohammed et al., Les bandes de jeunes

La Découverte | «Recherches »

2007 | pages 145 à 164

ISBN 9782707153456

Article disponible en ligne à l'adresse :

http://www.cairn.info/les-bandes-de-jeunes--9782707153456-page-145.htm

\section{!Pour citer cet article :}

Mathias Millet, Daniel Thin, «7. École, jeunes de milieux populaires et groupes de pairs », in Marwan Mohammed et al., Les bandes de jeunes, La Découverte « Recherches », 2007 (), p. $145-164$.

Distribution électronique Cairn.info pour La Découverte.

(C) La Découverte. Tous droits réservés pour tous pays.

La reproduction ou représentation de cet article, notamment par photocopie, n'est autorisée que dans les limites des conditions générales d'utilisation du site ou, le cas échéant, des conditions générales de la licence souscrite par votre établissement. Toute autre reproduction ou représentation, en tout ou partie, sous quelque forme et de quelque manière que ce soit, est interdite sauf accord préalable et écrit de l'éditeur, en dehors des cas prévus par la législation en vigueur en France. Il est précisé que son stockage dans une base de données est également interdit. 


\section{7 \\ École, jeunes de milieux populaires et groupes de pairs}

Mathias Millet et Daniel Thin

Existe-t-il un rapport entre les formes que prennent les sociabilités juvéniles des élèves de milieux populaires et les ruptures scolaires qui marquent la scolarité d'un certain nombre d'entre eux ? L'importance des relations de «quartier » chez ces élèves, des contradictions voire des oppositions, souvent notées par les sociologues, entre les logiques de ces liens et celles de l'école, permet-elle d'expliquer, comme on le croit parfois, les scolarités chaotiques ou avortées qui sont dix fois plus fréquentes chez les enfants d'ouvriers que chez les enfants de cadre [Broccolichi 1998] ? L'intensité et les formes des relations entre pairs sont-elles jouées avant tout rapport scolaire? Au contraire, les parcours scolaires heurtés et disqualifiants n'alimentent-ils pas les liens fondés sur des pratiques contraires non seulement aux exigences et à la morale scolaires, mais contraires aussi à la morale dominante et à l'ordre social ? Quelle place convient-il de faire aux parcours scolaires dans les rapports des élèves de milieux populaires à leurs pairs?

C'est à ces différents questionnements que sont consacrées les pages qui suivent, abordant le problème du rapport entre l'école, le quartier et la construction des solidarités contraignantes tramant les sociabilités juvéniles populaires. Appuyé principalement sur les résultats de deux recherches sur les parcours de ruptures scolaires de collégiens de milieux populaires [Millet-Thin 2005] et sur les classes relais [Kherroubi, Millet, Thin 2005], ce texte entend montrer le rôle central que remplissent l'école et les scolarités dans la détermination des relations et des pratiques de sociabilité des collégiens de milieux populaires avec leurs pairs. Il fait apparaitre que si la relation au groupe de pairs et au quartier peut être la source de tensions avec les exigences scolaires, l'école, par ses verdicts et ses classements négatifs, surdétermine l'importance des pairs (et notamment des pairs les plus « réfractaires » aux logiques scolaires) en rejetant du côté des pratiques les moins conformes les élèves qu'elle discrédite et qu'elle porte à se 
« valoriser » socialement et symboliquement ailleurs, selon d'autres logiques et d'autres pratiques.

\section{LES PAIRS, L’ÉCOLE ET LES DOUBLES CONTRAINTES}

\section{La force symbolique des pairs et des sociabilités de quartier}

Les sociabilités juvéniles constituent un aspect important de la vie sociale des collégiens de milieux populaires en ruptures scolaires que nous avons étudiés, qui se manifeste à la fois par l'attachement des collégiens aux relations du quartier d'origine, par l'affirmation d'un entre-soi rassurant et amical, par une socialisation mutuelle et le partage de références communes en matière de culture langagière, comportementale, musicale ou encore vestimentaire... Maintes fois évoquées lors des entretiens et maintes fois constatées lors d'observations réalisées dans les classes, les sociabilités juvéniles témoignent de la force symbolique exercée par les pairs dans la formation des catégories d'action et de perception des collégiens. Pour plusieurs d'entre eux, le groupe de pairs et, à travers lui, les relations liées au quartier d'habitation apparaissent comme une ressource où se nourrissent des liens de solidarité, de reconnaissance sociale et symbolique. Groupes de pairs et relations de quartier agissent à la fois comme réseau de sociabilité et comme éléments d'identification qui, pour être souvent socialement stigmatisants, n'en exercent pas moins des fonctions de socialisation.

Sébastien, par exemple, se caractérise par son ancrage et son implication dans les réseaux de sociabilité juvénile du quartier paupérisé et stigmatisé qu'il habite depuis sa naissance. Il insiste, au cours de l'entretien, sur l'importance de sa vie en bas des immeubles de son quartier et des relations qu'il entretient avec d'autres jeunes, parfois plus âgés que lui, souvent hors école et hors emploi. Sébastien reste rarement à l'intérieur de l'appartement. «Il n'aime pas trop rester enfermé, nous dit sa mère, c'est un gamin qui aime bien faire son petit tour, tourner, revenir. C'est pas un gamin qui va rester enfermé pendant une journée entière dans une maison hein, ah non, il faut qu'il voit du monde, qu'il bouge, qu'il rigole... [...] Dans mon quartier, je dis la vérité, il connaît tout le monde et puis tout le monde le connaît ». L'importance de cette sociabilité de quartier ${ }^{1}$ le conduit à refuser de s'éloigner pendant les vacances, par exemple pour participer à des activités organisées par les centres sociaux. Retrouvant l'idée selon laquelle «ce sont les plus vulnérables scolairement qui manifestent le plus d'anxiété à l'idée de partir sans leurs copains. » [Masclet 2001, p. 64], sa mère nous raconte ainsi : « tous les étés, c'est pareil, je lui

\footnotetext{
1. Qui n'est pas sans rapport avec la crainte d'affronter des univers socialement étrangers.
} 
dis : 'Il y a des activités, regarde, ils partent là dans un camp et tout, c'est pas cher'. Eh ben, il me dit non, il aime rester là, dans le quartier. Alors il traîne, il a rien à faire. »

L'influence des sociabilités de quartier se manifeste souvent en creux, lorsque l'histoire familiale et scolaire des collégiens les coupe de leur quartier d'origine. Dans les entretiens, les collégiens déracinés expriment leur nostalgie à l'endroit de leur ancien quartier, en général celui où ils ont grandi et sont allés à l'école élémentaire. Souvent, ils y retournent, notamment lorsque leur lieu d'habitation n'offre pas la possibilité de relations similaires à celles qu'ils ont connues, plus encore lorsque les aléas de la vie familiale ou les sanctions de l'institution scolaire conduisent les collégiens dans des collèges où les élèves de familles populaires sont minoritaires. Ainsi en va-t-il d'Amina qui, obligée de déménager avec sa mère, s'est trouvée contrainte de quitter son collège, son quartier, sa ville. Alors qu'au moment de l'enquête, aucun des agents institutionnels ne sait où Amina est scolarisée, l'entretien avec sa mère révèle qu'elle s'est réinscrite, à l'insu de cette dernière, dans un collège de sa ville d'origine où sont scolarisées ses amies. Durant l'entretien, Amina fait part de son regret de ne plus habiter cet endroit où elle voudrait retourner, son départ ayant coïncidé avec un déracinement social et affectif vécu d'autant plus douloureusement que sa scolarité dans son nouvel établissement ne se passe pas bien.

Les sociabilités de quartier offrent aux collégiens un ensemble de supports relationnels et symboliques dans lequel ils se valorisent et partagent de mêmes codes sociaux. La force symbolique des pairs réside ainsi dans un lien d'identification à des semblables et à de communes expériences du monde social. La séparation avec les amis d'enfance, partageant les mêmes manières d'être et les conditions d'existence propres aux quartiers paupérisés et disqualifiés, est vécue comme une rupture affective et un risque d'isolement social. De fait, les collégiens maintiennent tant bien que mal des liens avec leurs copains du " quartier » et rechignent à s'insérer dans de nouveaux réseaux de sociabilité quand leurs nouveaux voisins de résidence ou de collège appartiennent à d'autres catégories sociales ou sont davantage acculturés aux logiques scolaires.

Si la question des relations avec les pairs et de la place vis-à-vis des copains est présente chez tous à un moment ou à un autre de leur parcours, tous les collégiens rencontrés ne sont pas impliqués au même titre dans un réseau de sociabilité juvénile dense et tous n'opposent pas à l'école un groupe de pairs stable. Ainsi Clément a-t-il assez peu de copains avec lesquels il entretient des relations soutenues. En dehors de l'école, il reste souvent chez lui, devant la télévision ou sa console de jeux électroniques. Caroline est un peu dans la même situation. Néanmoins, tous deux parlent avec regret, et en le mythifiant, du quartier qu'ils ont quitté depuis plu- 
sieurs années, des relations et de l'«ambiance » qui y régnaient. Qu'ils soient ou non dans des réseaux de sociabilité juvénile dense, il reste que tous les collégiens interrogés se revendiquent (à un degré ou à un autre) de ce qu'on nomme parfois la " culture des rues » [Lepoutre 1997], c'est-àdire de styles musicaux, de pratiques langagières, de logiques comportementales..., qui sont à la fois issus des quartiers populaires et attribués à leurs jeunes habitants.

C'est ainsi que les pratiques langagières de plusieurs collégiens s'enracinent dans le langage " de la rue » où se mélangent des formes de verlan, des expressions portées par la musique rap, des anglicismes ou des expressions arabes "argotisés » et des formes d'un jargon local toujours réinventé. Sébastien et Christopher l'utilisent de manière ostentatoire au cours de l'entretien comme au cours de leurs pratiques langagières ordinaires. Le premier affirme son implication dans les rues de son quartier en mobilisant avec fierté des termes argotiques, par exemple pour désigner la police : «Parce que si dans la vie t'écoutes tout le monde, si tu te laisses faire ils te bouffent. Faut s'aider sans personne ni la police personne. Pas besoin des ch'nâres, de personne. (Qu'est-ce que c'est les ch'nâres ?) Les decs. » Le second revendique à la fois sa maîtrise du verlan et sa pratique du rap, y compris en « rappant » au cours de l'entretien : «(il reprend en chantant) 'Ambiance, scandale, danse de vandales, sens d'où vient la chaleur', voilà, DJ, breakeurs... [...] (Qu'est-ce que tu connais comme groupes ?) Là tout c'que j'vais vous dire elle s'ra finie la cassette. [...] Fonky Family, troisième œil, I AM, NTM... [...] Ouais '42160, (il rappe), ouais, je représente le trou du cul de la France, une p'tite cité'. Nous aussi on a un groupe au quartier ! [...] On rappe. On a une table de mixage. Ils l'ont payé super cher. Y en a qui z'ont cassé les diamants ! Les diamants c'est un truc dessus où on met et ça fait plus, ça fait plus... [...] (Tu sais scratcher, toi ?) Bien sûr j'sais scratcher! La dernière fois j'ai fait ça. J'fais du hip hop. [...] Faut pas oublier ça aussi ! Hein notre génération hein !»

De la même manière, les relations juvéniles des collégiens impliquent parfois l'adoption de postures agonistiques et belliqueuses, caractéristiques de la «culture des rues ». Ne pas s'y conformer risque de faire perdre la face devant les autres et d'affaiblir la place occupée dans le groupe de pairs. François l'explique clairement: «S'faire respecter aussi, parce que y a des élèves qui disent des mots qui te plaît pas et tout ! Ch'ais pas, ils arrivent vers toi, ils t'disent 'aïe [...] L'autre vers toi, il t'dit, l'autre, il t'a insulté'. Il va rapporter c'qui s'est passé, et toi, ça te plaît pas, t'aimes pas quand on parle derrière moi, ça t'fait une réputation. J'arrive vers celui qu'a dit ça, t'essaies de régler le problème et après, si l'autre aussi, il veut s'faire respecter et ben ça dégénère, après ça part en bagarre. » Se battre est une question d'honneur et de défense de sa place dans le groupe de pairs. 
Les activités que partagent les collégiens avec leurs pairs sont souvent des activités sans encadrement institutionnel ou associatif, qui privilégient le plaisir d'être ensemble sur des activités ayant des buts précis. « D'temps en temps on va en ville, d'temps en temps on joue au foot, on bouge, on sort, on traîne, la routine » (Tahar). Mehdi pour sa part explique : "J'me levais normal pour aller à l'école, et moi des fois j'rencontrais des copains sur la route, 'allez viens on va au squat', on allait dans un coin, quand on était petits, on traînait comme ça, on fabriquait des coins, on faisait des maisons, on montait dans les arbres comme les yamakasis et tout, on faisait tout (rires). " La rareté du recours à des institutions de socialisation secondaire qui tend à laisser les occupations des jeunes de milieux populaires sans encadrement autres que ceux de la « rue » [Chamboredon 1971] fait que leurs pratiques, plus souvent effectuées dans l'espace public (versus espace privé et protégé des regards extérieurs), ont une visibilité importante. Étant moins soustraites aux regards extérieurs, elles ont d'autant plus de chance d'être identifiées comme pratiques perturbatrices de l'ordre ou illicites. Karim explique comment le temps passé avec ses copains au bas des immeubles du grand quartier populaire qu'il habite conduit à une suspicion de "délinquance »: "Avant j'traînais tout l'temps avec eux mais maintenant j'traîne plus. J'traîne moins. (Et pourquoi ?) [...] On fait pas d'embrouilles, j'sais pas, j'aime pas... C'est l'regard des gens, ils vont dire 'ouais délinquants' j'sais pas quoi tout ça. »

La forte présence de plusieurs collégiens dans les rues avec leurs pairs n'est pas sans conséquence sur leurs pratiques qui flirtent parfois avec les "illégalismes » [Mauger 2001]. Elle peut conduire aussi à un repérage par les institutions chargées de contrôler et de maintenir l'ordre public, surtout quand les collégiens se retrouvent dans des lieux à des moments où ils ne devraient pas s'y trouver du point de vue des normes morales et éducatives dominantes. C'est le cas de Mehdi qui est plusieurs fois ramené chez lui tard le soir par la police : "Combien d'fois les flics me l'ont ramené euh, la police, mais j'suis trop mal ciblée par la police. [...] Parce qu'il traîne dans les rues, c'est pour ça je veux pas qu'il dorme à l'extérieur. Quand il dit qu'il dort chez Karim, il s'engueule avec lui, il lui dit 'allez, casse-toi' et il s'casse à une heure du matin parce qu'il est fier. [...] Et c'con au lieu d'rentrer à la maison directement, il va voir d'autres galériens qui traînent dehors et il traîne avec eux, il essaie de faire une nuit blanche. À deux heures du matin, on m'le ramène et on me dit il a pas fait d'bêtises, il a rien fait, il a pas cassé d'voitures, il a rien fait, mais il traîne dehors » (mère de Medhi). 


\section{De la double contrainte aux conflits de normes}

L'importance des sociabilités juvéniles n'est pas non plus sans effet sur les pratiques des collégiens au sein des établissements scolaires. Lors des entretiens, les collégiens en ruptures scolaires adoptent volontiers un ton jubilatoire pour décrire l'ambiance «animée » de leur classe, les chahuts et franches rigolades qui font les bons moments passés ensemble, y compris à propos des manières de perturber l'ordre scolaire ou de fuir l'espace de l'école : «Dans ma classe, on rigolait bien. [...] Avec un copain, on s'insultait. La prof, elle nous engueulait. Mais on f'sait semblant de s'insulter tous les deux. La prof ensuite elle pleurait à la fin (rire). [...] Elle en avait marre! Elle nous mettait tous les deux dehors. Chaque fois que t'es à côté d'une porte qu'on ouvrait les portes, on criait. Oh mais n'importe quoi ! (rire) » (Fabien).

Les relations des collégiens de milieux populaires avec les agents de l'institution scolaire présentent souvent les caractéristiques d'une opposition entre "Eux», autrement dit les agents de l'institution scolaire auxquels peuvent être associés les « intellos » ou les « bouffons », c'est-à-dire les élèves adoptant les postures scolaires conformes, et « Nous », c'est-àdire l'ensemble de ceux qui, connaissant les mêmes conditions d'existence, partagent des postures scolaires hétérodoxes. Cette opposition traverse les discours de tous les collégiens rencontrés, y compris de ceux dont l'insertion dans un groupe d'élèves réfractaires aux règles scolaires est faible comme Caroline qui, selon ses termes, marque ses distances aux « bourges » qui « fayotent »: " Il y en a qui font semblant d'être des petites bourges parce qu'ils se prêtent leurs habits entre eux. [...] Ils sont vraiment lèche-bottes ceux-là. [...] Dans la cour, ils insultent et ils se moquent des profs. Et tandis que quand ils sont pendant les cours, ils sont vraiment lèche-bottes... Y a un prof qui va dire 'qui peut aller me chercher des cahiers?' ; ils vont tous lever le doigt pour partir [...] Et puis quand ils sont dehors ils se moquent toujours des professeurs. "

Les relations entre pairs passent ainsi par des signes de reconnaissance mutuelle. Pour bénéficier des profits symboliques afférents à la reconnaissance des pairs, les collégiens doivent se faire reconnaître par leurs pratiques et leurs postures comme étant des leurs. Cette exigence pousse Rochdi, arrivant en cinquième dans un nouveau collège, à jouer le rôle du perturbateur de la classe, d'abord discrètement comme s'il ne voulait pas s'aliéner d'emblée les enseignants, puis de manière plus ostensible, jusqu'à l'exclusion définitive trois mois après son entrée dans l'établissement. "Les autres étaient ravis des problèmes qu'il causait en classe puisque c'était des distractions qui permettaient de pas avoir cours. [...] J'ai d'ailleurs toujours eu l'impression que c'était pour ça qu'il le faisait quoi, c'était une manière de se faire aimer des autres aussi cette manière de tou- 
jours créer des problèmes en classe, parce que ça amusait les autres » (professeur d'anglais).

Cette description des relations de sociabilités juvéniles des collégiens en ruptures scolaires et de leurs pratiques hétérodoxes semble accréditer l'idée selon laquelle leur intensité et leur forme socialiseraient, en amont et en dehors de la scolarisation, ces enfants de familles populaires à une culture anti-école responsable des "désordres scolaires》 et des parcours scolaires chaotiques ou de "déscolarisation». Le groupe de pairs et la sociabilité juvénile seraient alors premiers dans le processus, et les ruptures scolaires signeraient l'inscription symbolique ou réelle des jeunes de milieux populaires dans la «culture des rues » et, pour certains, dans la délinquance. Ainsi, l'opposition aux institutions s'engendrerait prioritairement ou exclusivement dans des processus externes et étrangers à la scolarisation elle-même. De même, le sens commun pourrait-il en sortir renforcé selon lequel les ruptures scolaires, sous-tendues par l'intensité de la relation aux pairs, seraient sources d'activités délictuelles et d'illégalismes, bref de délinquance. Pourtant, aucune étude ne permet pas de lier ruptures scolaires, sociabilités juvéniles et délinquance. Synthétisant les résultats du programme de recherches sur la «déscolarisation», Dominique Glasman rappelle que «le lien entre déscolarisation et délinquance n'y est pas établi » et que, contrairement aux représentations communes, «Une part des déscolarisés vit plutôt dans l'isolement si ce n'est l'enfermement à domicile, une autre part se trouve embarquée dans le travail dans un cadre familial ou communautaire, 'trainent' sans pour autant se livrer à des actes répréhensibles », pour enfin conclure que «si les délinquants ont souvent été des jeunes qui ont quitté précocement l'école, en revanche les déscolarisés sont loin d'être tous et de devenir fatalement délinquants » [Glasman, CEuvrard 2004, p. 63-64].

Surtout, la recherche conduit à interroger fortement l'opinion selon laquelle l'intensité symbolique et pratique de la relation aux pairs serait jouée d'emblée, avant et en dehors de toute expérience scolaire, et serait première dans le processus conduisant les collégiens aux ruptures scolaires ou à l'adoption de comportements a-scolaires. Certes, il est acquis que la relation au groupe de pairs et au quartier participe d'une tension avec les exigences scolaires et institutionnelles, ne serait-ce qu'au regard des écarts grandissants entre les exigences scolaires et ce que vivent les collégiens au sein de familles et de quartier aux conditions d'existence dégradées. Les ruptures scolaires s'enracinent en effet dans les tensions qui trament la scolarisation des enfants de classes populaires, tensions qui s'inscrivent d'abord dans la confrontation entre les logiques familiales et scolaires [Thin 1998]. Elles se prolongent ensuite dans les solidarités contraignantes issues de la relation avec les pairs. Associée aux nécessités scolaires, celleci forme une double contrainte, c'est-à-dire une configuration dans la- 
quelle les collégiens sont en proie à des exigences contraires, voire antinomiques ${ }^{2}$. L'ancrage dans le quartier et les complicités solidaires du réseau de sociabilité juvénile concourent ainsi à une sorte de résistance à l'acculturation et à la socialisation scolaires, faisant passer pour normales des pratiques non conformes voire réfractaires à l'ordre scolaire, qui s'objective dans les pratiques hétérodoxes des collégiens. Cependant, loin de se traduire toujours et dès l'abord en opposition avec l'institution scolaire et ses agents ${ }^{3}$, c'est au fil des déconvenues et des malheurs scolaires, que ces tensions tendent à se transmuer en conflits de normes entre collégiens et agents de l'institution scolaire.

Il en est ainsi des questions des dissonances langagières qui sont au cœur des ruptures scolaires ${ }^{4}$. Lorsque s'aggravent les parcours scolaires des collégiens, ce qui n'était au départ que dissonances langagières, se cristallise en conflits de normes linguistiques [Lepoutre 1997] autour des usages langagiers socialement acceptables. Les collégiens manifestent un fréquent rejet symbolique de la langue scolaire qui puise son origine dans la tension entre le langage scolaire et le langage de la socialisation primaire des collégiens. Pour autant, ce rejet n'apparaît pas d'emblée. Il se construit et se renforce avec la difficulté cognitive à maîtriser la langue scolaire, ses règles et ses exigences. Il s'accentue et devient résistance à mesure que la disqualification scolaire des collégiens progresse et les repousse du côté des codes sociolinguistiques juvéniles les plus éloignés des normes scolaires. Dans une sorte de retournement du stigmate scolaire, certains collégiens en viennent à dire leur hostilité vis-à-vis de certains professeurs qui «se la jouent» en employant des "mots compliqués » ( ( C'est pas qu'on comprend pas mais c'est nul, c'est pour s'la jouer...») ou vis-à-vis d'autres élèves dont le langage plus châtié leur fait dire qu'ils emploient des «mots de bourges». De même, ils disent parfois l'impossibilité dans laquelle ils sont d'employer des mots ou des expres-

${ }^{2}$. Dans ce sens, il existe de fortes analogies entre les collégiens en ruptures scolaires dont nous avons étudié les parcours et les lycéens et étudiants étudiés par Stéphane Beaud [Beaud 2002] du point de vue de la tension entre l'ancrage dans la sociabilité des quartiers populaires et les exigences de la scolarisation.

${ }^{3}$. D'ailleurs, on sait que tous les élèves de milieux populaires qui vivent ces doubles contraintes ou ces tensions entre socialisation scolaire et formes de socialisation populaire n'entrent pas dans un parcours de ruptures scolaires ou de défiance vis-à-vis de l'école. Les élèves de milieux populaires qui accomplissent une scolarité " réussie » vivent ces contradictions mais parviennent à les résoudre au profit de leur scolarité (ce qui d'ailleurs ne signifie pas que les tensions disparaissent) et parfois au prix d'un éloignement des pairs et de leur sociabilité, que cela soit une conséquence de l'investissement scolaire ou pour éviter de se compromettre dans des situations illégitimes [Bruneteaux 1994].

${ }^{4}$. La recherche montre en effet que les difficultés d'apprentissage scolaire de ces collégiens sont intimement liées à la difficile conversion des structures sociolinguistiques issues de la socialisation primaire au sein des familles populaires en structures sociolinguistiques scolaires (conversion qui relève d'une conversion socio-cognitive) [Bernstein 1975; Lahire 1993]. 
sions qui leur paraissent étrangers à eux-mêmes et dont l'usage est ressenti comme une trahison symbolique du monde de leur sociabilité ordinaire ${ }^{5}$.

\section{LES PAIRS COMME CAPITAL COMPENSATOIRE À LA DISQUALIFICATION SYMBOLIQUE}

Si les jeunes des quartiers populaires se socialisent, aux manières de faire et de parler, aux pratiques et aux normes du groupe des « jeunes de la cité » [Bordet 1998] et de la «culture des rues », ce n'est pas seulement parce qu'ils partagent de mêmes espaces d'existence, de mêmes conditions de vie, de mêmes assignations dans l'espace social. Leur adhésion aux formes de la sociabilité juvénile propres aux quartiers populaires se construit en interrelation avec la socialisation scolaire qui, pour eux, se confond avec l'expérience souvent précoce des difficultés scolaires et des conflits avec l'institution scolaire. Le rapport des élèves de milieux populaires en ruptures scolaires au groupe de pairs les moins soumis aux logiques scolaires, comme les tensions qui en résultent dans le rapport à l'école, se comprend ainsi à partir de leur expérience scolaire.

\section{Des tensions scolaires au désordre scolaire}

Les pratiques les moins conformes à l'ordre scolaire, qui perturbent et entravent l'activité pédagogique et souvent transgressent l'ordre moral porté par l'école, ne peuvent s'expliquer simplement par la pression collective des pairs sur les collégiens qui se verraient enjoints de multiplier incartades, rebuffades ou de résister à l'autorité pédagogique [Thin 2002]. Les pratiques perturbatrices de l'activité pédagogique, loin d'être réductibles à la seule manifestation plus ou moins ludique ou hostile de pratiques sociales juvéniles contraires à l'ordre scolaire, sont aussi le résultat des difficultés d'apprentissage des collégiens et plus généralement des décalages entre leurs postures dans la classe et les attentes scolaires. Tels sont ces collégiens qui interrompent sans cesse l'enseignant dans une tentative désespérée de " raccrocher » au cours par un questionnement incessant et «hors de propos » ou qui, ne parvenant pas à suivre, s'agitent et s'énervent, et finissent parfois par affronter l'enseignant qui, lassé d'être interrompu, refuse de répondre à leurs questions décalées. De même, les

\footnotetext{
${ }^{5}$. D'un autre côté, le langage utilisé par les collégiens dans la classe et au cours des interactions ordinaires s'écartant des normes linguistiques des enseignants (mixtes de normes scolaires et de normes liées à leur catégorie sociale) génère chez ces derniers un mélange d'incompréhension et d'indignation morale. Les cris ou les interpellations sonores entre collégiens, les insultes rituelles, le vocabulaire mobilisé heurtent l'ethos des enseignants qui y voient vulgarité et violence. Les usages langagiers des collégiens, qui résonnent comme une offense aux convenances linguistiques et éthiques des enseignants, font ainsi l'objet de réprobations morales qui s'ajoutent aux enjeux strictement scolaires d'acculturation au français standard.
} 
absences et les pratiques multiples d'évitement de la contrainte scolaire, qui peuvent être analysées comme des tentatives souvent désespérées pour se soustraire aux situations pédagogiques vécues comme potentiellement stigmatisantes, croisent souvent la priorité donnée par les collégiens aux sociabilités juvéniles sur les obligations scolaires en même temps qu'elles font suite à la multiplication des déboires scolaires. Il en est ainsi des absences d'abord ciblées sur les matières où s'accumulent les difficultés les plus criantes, puis généralisées à l'ensemble des cours, la situation scolaire étant devenue pour les collégiens insupportable et la présence en classe ne pouvant tourner qu'à l'affrontement avec les enseignants. Tel est le cas de ce collégien qui se fait volontairement exclure de la classe pour échapper aux heures de cours dans lesquelles il est scolairement perdu ou de tel autre qui « sèche » systématiquement un cours de langue pour prévenir les conflits dans lesquels il est enfermé avec l'enseignante concernée.

Les conflits avec les enseignants et les autres agents de l'institution scolaire sont bien souvent également le produit d'une dégradation scolaire amorcée parfois dès l'école primaire et précipitée avec l'entrée au collège. L'accumulation des malentendus cognitifs [Bautier, Rochex 1997], des sanctions négatives et des postures non conformes des collégiens est au principe d'engrenages qui les conduisent à affronter les enseignants selon des modalités contraires aux règles et à la morale scolaires et propres aux modes de relation juvénile dans les milieux populaires. Ces conflits, dont certains deviennent circulaires, alternant sanctions et représailles, mettent face à face les logiques contradictoires des collégiens et des enseignants en matière de régulation des comportements. D'un côté, les enseignants, qui tentent de sauvegarder le cadre de l'activité scolaire et leur autorité pédagogique, attendent que les conflits se règlent dans le respect des règles scolaires. De l'autre, les collégiens se trouvent fréquemment pris dans les contraintes du groupe de pairs qui imposent de ne pas perdre la face et de "sauver son honneur ». L'importance qu'il y a, pour ces collégiens, à défendre « leur honneur » renvoie à leur relative dépossession au plan matériel comme au plan symbolique ${ }^{6}$. Dominés dans l'ordre des logiques scolaires, ces collégiens peuvent tenter alors de donner un tour différent aux conflits qui les opposent aux enseignants en amenant ces derniers sur le terrain de la logique agonistique du défi. Du coup, lorsqu'un enseignant s'emporte et sort du registre de rôle attendu par sa fonction qui suppose d'agir selon des règles impersonnelles de maîtrise de soi et non selon ses humeurs [Vincent 1980], il favorise le déplacement du conflit sur le registre d'un affrontement, dont les formes se rapprochent des modalités pro-

\footnotetext{
${ }^{6}$. «Quant au jeune de banlieue (...) il a sans doute un sentiment exacerbé de l'honneur parce qu'il ne possède rien et n'a rien d'autre à défendre que la présentation de soi-même comme dirait Erving Goffman : il est le dos au mur. Il ne peut développer des stratégies très sophistiquées pour défendre son honneur " [Castel, Haroche 2001, p. 60-61].
} 
pres aux conflits entre collégiens eux-mêmes, où ce qui est en jeu est de ne pas perdre la face.

Ce qui n'était au départ qu'un ensemble de tensions sociales et cognitives dans les postures langagières, corporelles, les normes de maîtrise de soi, etc., se transforme, au fil du parcours de disqualification, en conflits de loyauté entre allégeance au groupe de pairs et allégeance à l'enseignant. La question qui se pose est donc moins celle du primat des logiques et des sociabilités juvéniles populaires dans la production de pratiques d'opposition à l'école que celle des conditions du passage de sociabilités et de logiques juvéniles potentiellement en tension avec certaines exigences scolaires, et en tension d'abord parce qu'inscrites dans la confrontation des logiques socialisatrices familiales avec le mode scolaire de socialisation, à une influence déterminante des pairs pouvant prendre la forme radicalisée de la culture de provocation et de la culture anti-école.

\section{Disqualification scolaire et refuge symbolique des pairs}

Pour prendre toute la mesure du poids de la scolarisation dans la définition des relations avec les pairs, et singulièrement les pairs les plus réfractaires à l'ordre scolaire, il convient de considérer les effets symboliques de la scolarisation sur les élèves de milieux populaires qui ne parviennent pas à satisfaire aux exigences scolaires et que l'école échoue à faire accéder à ses savoirs. Comme le dit Jean-Claude Passeron, on ne saisit jamais entièrement les enjeux de la formation et de la certification scolaires qu'en tenant compte des effets sociaux de leur «alchimie symbolique », irréductibles aux aspects économiques [Passeron 1982, p. 578]. À rebours de la qualification symbolique produite par l'accès au diplôme, les sanctions négatives de la scolarité sont aujourd'hui ${ }^{7}$ génératrices d'une profonde disqualification symbolique [Millet, Thin 2004].

Les collégiens en ruptures scolaires dont nous avons étudié les parcours, comme bon nombre d'enfants issus des fractions inférieures des classes populaires, cumulent les difficultés dans les apprentissages scolaires, souvent depuis l'école élémentaire ${ }^{8}$. L'accumulation des difficultés d'apprentissage, des contre-performances scolaires et des sanctions négatives qui les accompagnent, loin de laisser les collégiens indifférents, est

${ }^{7}$. « Aujourd'hui » parce que la scolarisation a pris une part de plus en plus importante non seulement dans la distribution des positions et des places dans l'espace social mais aussi dans la qualification des individus sur les différents marchés des échanges économiques et des échanges symboliques.

${ }^{8}$. S'il n'est pas utile de revenir ici sur les explications sociologiques de cette prégnance des difficultés scolaires dans les milieux populaires mises au jour par de nombreuses recherches, il importe de souligner, en revanche, contre une interprétation qui réduirait les parcours de ruptures scolaires à un refus de l'école ou à des comportements inadéquats, que les difficultés dans les apprentissages scolaires trament tous les parcours étudiés. 
porteuse de stigmatisation et de sentiment d'indignité scolaire et culturelle [Bourdieu, Passeron 1970], que les collégiens manifestent dans les entretiens par leur refus de parler de leurs résultats ou en affirmant leur sentiment d'incompétence scolaire («je suis nul »). C'est un processus de stigmatisation et d'auto-stigmatisation, c'est-à-dire d'intériorisation du stigmate, qui s'enclenche au long de la scolarité et qui est au principe de nombre de pratiques contraires à l'ordre scolaire.

La dévalorisation de $\operatorname{soi}^{9}$ et la stigmatisation, pour être d'abord scolaires, ne se limitent pas aux dimensions scolaires de l'existence. La tendance à l'universalisation des jugements et des verdicts scolaires, comme appréciation d'une grande partie de la personne, a ainsi pour effet de discréditer plus largement les élèves en difficulté, avec en plusieurs cas, des retentissements négatifs sur la place occupée dans l'espace des relations familiales. Il en va ainsi de ces collégiens qui, condamnés au sein de l'univers familial pour leurs différents manquements scolaires et les désordres familiaux qu'ils occasionnent, subissent une double disqualification : scolaire et familiale. Le capital scolaire négatif tendant à devenir un capital symbolique négatif au sein même de la sphère familiale, des lignes de clivage se dessinent parfois au sein des fratries. Une collégienne explique comment ses comportements scolaires contribuent à la disqualifier au plan des relations familiales, notamment par rapport à sa sœur scolairement plus performante. Soit encore ce collégien qui se bat avec son frère cadet scolairement plus doté comme pour sauver son honneur et laver ce qu'il perçoit, dans la concurrence avec celui-ci, comme une indignité personnelle. Non seulement, « les classements scolaires redistribuent les cartes au sein des familles et bousculent les hiérarchies symboliques dans la fratrie » [Beaud 2002, p. 64], mais ils créent, dans les cas présents, une sorte de fracture scolaire au sein des fratries. La double disqualification symbolique, scolaire et familiale, réduit alors les supports [Castel, Haroche 2001] que les collégiens peuvent trouver pour renverser le cours de leur scolarisation et plus largement pour établir une place sociale et symbolique positive dans le monde social.

Pour les collégiens en ruptures scolaires, l'école, par ses verdicts et ses classements négatifs, devient un lieu d'insécurité ${ }^{10}$ symbolique, chaque moment de la vie scolaire devenant porteur d'un risque de discrédit et de conflits avec les enseignants. L'insécurité symbolique se décèle par exemple chez un collégien réputé perturbateur de l'ordre de la classe et dont on

${ }^{9}$. Elle conduit souvent les collégiens à renoncer à toute tentative d'apprentissage, parfois à un refus explicite des tâches scolaires (que ce soit en disant « je ne sais pas faire » ou en disant " je ne veux pas faire »), et peut entraîner des manifestations corporelles d'agitation ou à l'inverse d'apathie, sortes de tactiques de fuite ou d'évitement non-conscientes.

10. Au sens où les collégiens sont dans une forte incertitude ou une forme de précarité quant à leur avenir scolaire, quant aux profits scolaires qu'ils tireront de leur scolarité et même quant à leurs relations quotidiennes avec les agents de l'institution scolaire. 
apprend, à l'occasion de l'entretien réalisé avec sa mère, que la confrontation aux exigences du collège provoque chez lui insomnie et même réactivation d'une énurésie infantile. Elle se manifeste dans les pratiques d'un autre, à la réputation de «caïd », qui fuit toutes les situations risquant de révéler ses difficultés avec l'écrit scolaire. Plus la situation scolaire se dégrade, et plus le groupe des pairs partageant cette disqualification scolaire, en même temps que des pratiques, des manières de faire ou de parler contraires aux normes scolaires, tend à devenir un refuge et un rempart symboliques dans l'espace scolaire comme en dehors. Sorte de capital relationnel compensatoire de l'absence de reconnaissance scolaire, le groupe de pairs devient rempart quand il permet de trouver des appuis pour faire face aux sanctions des agents de l'institution scolaire ou quand il offre des justifications aux pratiques les moins scolaires.

L'affiliation aux pairs les plus éloignés des normes scolaires est aussi refuge au sens où les collégiens y trouvent des formes de reconnaissance là où la scolarisation produit du discrédit. Ainsi Clément souligne que ses conflits avec les enseignants et les responsables du collège lui attirent une forme de notoriété parmi les pairs compensant sa solitude scolaire : «vu que quand y a quelqu'un qui frappe les surveillants ou surtout la principale, tout le monde se fait connaître hein. Moi je les connaissais tous. À chaque fois que je les vois ils me serrent la main, les filles elles me font la bise et tout ». Les solidarités juvéniles peuvent trouver ainsi à se construire contre les agents de l'institution scolaire. La solidarité aux pairs (dont ils partagent les conditions, les difficultés, le quartier) qui rassurent et protègent du monde (hostile et stigmatisant) des "Autres", et l'adoption à l'école de certains aspects de « la culture de provocation » [Beaud, Pialoux 2003] par les collégiens sont alors l'envers du décor scolaire de leur dépréciation symbolique et sociale. Elles répondent à la nécessité symbolique de mettre en place des contre-handicaps [Grignon, Passeron 1989] sociaux pour faire face tant bien que mal à l'accumulation des « handicaps » scolaires.

Aussi la double contrainte évoquée plus haut entre logiques juvéniles et logiques scolaires, plus difficile à soutenir pour les moins armés face aux exigences scolaires, se résout-elle au profit de l'inscription dans les pratiques et manières d'être des pairs les moins conformes aux normes scolaires à mesure que se renforce la disqualification symbolique engendrée par la scolarisation et l'officialisation de la rupture scolaire. Plus les stigmates scolaires se multiplient, plus la disqualification symbolique à l'école s'accroît, et plus les collégiens sont aspirés par la sociabilité juvénile des pairs les moins soumis aux logiques scolaires. Ce rôle de refuge symbolique peut se jouer au sein du collège ou de la classe par la valorisation auprès des pairs, via des pratiques de perturbations de l'ordre pédagogique ou de contestation de l'autorité professorale, en particulier aux moments 
où la disqualification scolaire devient publique. Il peut se jouer par l'aspiration des collégiens vers l'extérieur de l'espace scolaire, multipliant les absences et parfois les conflits à la périphérie de celui-ci. Enfin, le groupe de pairs remplit d'autant plus ce rôle de sécurité ou de refuge que l'école, loin d'apporter les satisfactions attendues, alimente au contraire un sentiment d'injustice et que celui-ci se greffe à des relations intrafamiliales dégradées. Ce faisant, les collégiens sont encore un peu plus renvoyés du côté des pratiques antinomiques avec l'ordre scolaire et de ceux qui les portent, l'effet de socialisation exercé en retour par les pairs les séparant encore un peu plus des normes et des règles scolaires comme de la scolarisation.

\section{L'assignation institutionnelle à la « déviance "}

Au-delà, l'apparentement des collégiens à ceux de leurs pairs que les institutions de socialisation et d'encadrement perçoivent comme porteurs de danger pour l'ordre social et pour eux-mêmes, est le produit des assignations institutionnelles qui résultent des catégorisations socio-scolaires émaillant les parcours de ruptures scolaires des collégiens. Les actes institutionnels peuvent concourir à rapprocher les collégiens des plus rétifs à l'ordre scolaire, que ce soit par les sanctions répétées et cumulées qui assimilent les collégiens sanctionnés au groupe des indociles $»{ }^{11}$ ou par les regroupements dans des espaces ou des dispositifs spécifiques pour les collégiens "réfractaires ». On peut évoquer l'exclusion collective de plusieurs collégiens, la désignation de certaines classes comme classes où règne le désordre ou encore les orientations vers les SEGPA ou les dispositifs relais qui, en regroupant des collégiens en ruptures scolaires, peuvent avoir comme effet (pervers) de renforcer l'intensité de la socialisation entre pairs. Ainsi, dans un collège, plusieurs collégiens accumulent les heures de retenue qui les réunissent chaque mercredi. Progressivement, ce temps partagé devient un temps où se développent les pratiques que condamnent l'institution scolaire et au cours duquel les collégiens aiment à se retrouver, au grand dam des responsables de l'établissement. En plusieurs cas, les mesures prises à l'encontre de collégiens (exclusions répétées ou prolongées par exemple), conduisent ces derniers à fréquenter plus assidûment la rue et les jeunes qui y séjournent. Ainsi, Rochdi a des horaires de plus en plus allégés et passe le plus clair de son temps dans la rue, le logement familial où cohabitent difficilement trois générations ne lui laissant guère d'espace propre.

Au long des parcours scolaires des collégiens, se met en place un processus de catégorisation institutionnelle qui s'échafaude à partir de leurs

\footnotetext{
${ }^{11}$. D'indocilis : que l'on ne peut instruire.
} 
postures corporelles, de leurs pratiques langagières, de leurs manières d'interagir avec les enseignants et avec les autres élèves mais aussi de la doxa dominante à propos des jeunes de milieux populaires. Ainsi, les discours sur la violence dont seraient porteurs ces derniers sont au principe de perceptions de leurs pratiques ou de leurs attitudes comme potentiellement menaçantes : " on le sent prêt à aller au contact ", "son regard est hostile $» .$. . Ces qualifications négatives, répétées et consignées dans les différents dossiers des collégiens qui finissent par remplir la fonction de casiers scolaires, participent d'une sorte d'assignation à la « déviance » scolaire et plus largement sociale. Elles alimentent la suspicion à l'endroit des collégiens et mettent à mal la présomption d'innocence scolaire face aux événements qui émaillent la scolarité de ces derniers. De même, les caractéristiques sociales (familiales, résidentielles...) des collégiens interprétées à la lumière des qualifications scolaires négatives par les agents de l'institution scolaire tendent à être renvoyées aux collégiens comme reproche, comme qualification négative a priori ou comme potentialité de désordre. C'est le cas lorsqu'un collégien arrivant dans un nouveau collège (après de nombreux déboires dans son précédent établissement) se voit rappelé le quartier et le collège d'où il vient pour lui signifier que les pratiques et les manières d'être qui leur sont associées n'ont pas cours dans le nouvel établissement : «On n'est pas à Vaulx-en-Velin ici ! » Le lieu de résidence et les groupes d'appartenance discrédités et discréditants tendent à devenir assignation institutionnelle aux pratiques " déviantes », assignation qui, ne laissant guère d'échappatoire, fonctionne alors comme force de rappel « au quartier » ou « aux pairs ». L'ensemble de ces actes, comme les actes d'officialisation institutionnels de la rupture du lien scolaire (mise à pied, conseil de discipline, exclusions provisoires et définitives) et la prise en charge par des dispositifs chargés des " anormaux d'école », peuvent ainsi contribuer à rejeter les collégiens du côté du capital relationnel des pairs, des pratiques les moins conformes et de la « culture des rues».

\section{PRATIQUES JUVÉNILES ET SCOLARISATION : UNE RELATION DIALOGIQUE}

Plus qu'elle n'est susceptible d'expliquer les ruptures scolaires, l'importance symbolique des pairs trouve à s'affermir dans le cadre de parcours de ruptures scolaires qui, par l'effet négatif des déclassements scolaires, et dès lors qu'ils se greffent à des difficultés familiales, portent les collégiens les plus discrédités à chercher à se valoriser socialement et symboliquement ailleurs ou autrement qu'en l'école. La variation du rapport aux pairs ne se comprend donc que si l'on tient simultanément compte de la situation et de l'expérience scolaires des collégiens, notamment du sentiment de valorisation ou au contraire d'indignité généré par la scolarisation. La place de l'école est essentielle dans la mesure où les modalités 
et le contenu (culturels) des relations entre pairs s'enracinent dans le déficit symbolique creusé par l'école et trouvent largement à se définir en contrepoint du scolaire. En repoussant par ses verdicts négatifs les collégiens les moins armés du côté des pratiques les moins conformes, l'école tend à surdéterminer l'importance symbolique des pairs, et d'abord les plus réfractaires d'entre eux. L'analyse de l'ensemble des parcours de ruptures scolaires permet ainsi de conclure que ce sont les processus scolaires, greffés aux processus familiaux, qui priment, la valorisation de soi dans les pratiques les moins conformes auprès des pairs les plus « réfractaires » apparaissant alors comme seconde dans la genèse des parcours de ruptures scolaires.

Pas plus qu'il ne s'agit d'un processus autonome et extérieur à l'école, l'affiliation aux pairs porteurs des pratiques assimilées à la "culture des rues " ne relève d'un processus en deux temps, avec une première phase débouchant sur ce que Sylvain Broccolichi appelle une «désaffiliation scolaire », et conduisant à une deuxième phase qu'il nomme "réaffiliation para-scolaire » [Broccolichi 1998, p. 46]. Il s'agit plutôt d'une tension inhérente à la scolarisation des enfants des classes populaires entre participation scolaire conforme aux exigences et aux règles scolaires et socialisation juvénile des pairs partageant les mêmes conditions d'existence et les mêmes dispositions, tension qui se résout dans le cas des collégiens étudiés "en faveur» de la sociabilité juvénile populaire. L'attrait des pairs et les conflits de normes qui sont à l'œuvre dans les parcours de ruptures scolaires apparaissent moins comme des causes de ces parcours que comme des constructions concomitantes contribuant à leur radicalisation. On peut parler d'une dialectique ou d'une relation dialogique entre le pôle de la scolarisation et le pôle des pairs et du quartier de référence. L'importance respective des deux pôles oscille et varie en fonction de ce que chacun des deux pôles apporte sur le plan social et symbolique aux élèves de milieux populaires et principalement en fonction de la valorisation ou de la stigmatisation scolaire.

L'interdépendance entre sociabilités juvéniles et scolarisation conduit à relativiser les thèses qui envisagent la «culture des rues » ou «des bandes » comme réalité symbolique autonome. En insistant sur l'imbrication des processus scolaires et des formes des sociabilités juvéniles populaires, on pointe la réduction qui est opérée chaque fois que l'interprétation autonomise complètement les pratiques et manières d'être regroupées sous le concept de «culture des rues » pour en faire le produit d'une socialisation endogène aux quartiers populaires. Il faut au contraire dire les liens d'interdépendance existant entre les formes que prennent les apparentements juvéniles des collégiens de milieux populaires en ruptures scolaires et le sens (comme direction et signification) de l'expérience que ces derniers font de l'école et de la scolarisation. La relation des collégiens de 
milieux populaires en ruptures scolaires à leurs pairs, et la force symbolique de ces derniers, loin d'être jouées d'emblée, trouvent à se construire dans le cadre d'une histoire sociale, et notamment scolaire, de disqualification. Par conséquent, elle ne saurait être interprétée indépendamment des effets de stigmatisation qui enferment les "victimes ", socialement désignées, des verdicts scolaires dans le cercle vicieux de "l'échec». Ces processus ont évidemment des effets sur l'économie psychique et relationnelle des collégiens, sur le rapport des élèves aux apprentissages, à l'institution, aux agents de l'institution, sur l'idée qu'ils se font d'euxmêmes en tant qu'élève et au-delà. $\mathrm{Si}$ le groupe de pairs constitue un refuge symbolique, il n'est pas pour autant indemne des effets des classements scolaires. Comme Hugues Lagrange le souligne, on ne peut suivre ceux qui, comme John Hagan, affirment « que la délinquance s'appuie sur une sous-culture qui permet aux jeunes de s'affranchir des normes scolaires et sociales et leur donne de l'assurance et de la fierté » [Lagrange 2001, p. 287]. Nos observations montrent au contraire que les pairs les plus réfractaires au scolaire et parfois les plus engagés dans les illégalismes témoignent d'un fort sentiment de disqualification par l'école ${ }^{12}$. Elles révèlent que les classements et les verdicts scolaires continuent à agir jusqu'au sein des groupes de pairs et peuvent avoir des effets symboliques sur la place des collégiens parmi les pairs qui, même s'ils partagent des logiques contraires aux exigences scolaires, $n$ 'en ont pas moins intériorisé la légitimité des hiérarchies scolaires. Ainsi, le stigmate scolaire peut être mobilisé par les copains lorsque les «vannes»s'appuient sur le niveau scolaire de l'un d'entre eux ou lorsqu'ils disqualifient sa parole au nom de sa place scolaire : " qu'est-ce que t'en sais toi t'es encore en cinquième ». Le fait que les hiérarchies scolaires participent, pour une part, aux relations et aux hiérarchies entre pairs, confirme l'impossibilité d'analyser les sociabilités juvéniles et la «culture des rues » comme des entités indépendantes de la scolarisation.

De même, l'attrait des pairs ne peut être pensé en dehors des effets de scolarisation liés à la place prise par l'école dans l'opération d'allocation statutaire et symbolique des individus (qualifiante pour les uns, infamante

\footnotetext{
${ }^{12}$. Les processus de disqualification scolaire n'agissent bien entendu pas seuls pour produire les pratiques des jeunes de quartiers populaires. Il faudrait encore pouvoir décrire, pour les prendre en compte, les processus de disqualification qui leur sont adjacents : la disqualification résidentielle liée au fait de vivre dans des quartiers dégradés, assimilés aux dangers sociaux ; la disqualification par l'origine " ethnique " dont sont victimes une partie importante des jeunes des quartiers populaires; la disqualification familiale qui, au delà des " problèmes scolaires ", sont liées au chômage et à la précarité que vivent une large partie des familles auxquelles est adressé en sus le reproche des pratiques de leurs enfants (comme on peut le voir dans le « contrat de responsabilité parentale » mis en avant par le gouvernement après les révoltes urbaines de l'automne 2005)... Il reste que la dimension scolaire est incontournable. Elle s'articule aux autres dimensions sociales de disqualification pour expliquer la « rage » qui périodiquement explose dans les quartiers populaires.
} 
pour les autres, et d'abord pour les fractions inférieures des milieux populaires situées du mauvais côté de la statistique scolaire). En cela, la question des sociabilités juvéniles populaires ne peut être dissociée de la nouvelle centralité de l'école, tant sur le plan de la construction des destinées sociales des individus que sur celui de la définition des attributs symboliques de la personne. Elle ne peut être dissociée non plus de la progression, jusque parmi les catégories sociales les moins scolarisées, de l'importance de l'école ${ }^{13}$, de la légitimité de ses classements et de l'élévation des aspirations scolaires. Elle doit être replacée dans le cadre de la massification scolaire qui, loin d'avoir assuré aux enfants des milieux populaires un accès égal aux savoirs et aux titres scolaires, a déplacé le lieu de construction des classements sociaux vers l'école, où s'opère " la reproduction des hiérarchies sociales par la transmutation des hiérarchies sociales en hiérarchies scolaires » [Bourdieu, Passeron 1970, p. 186]. Si, dans les années 1950, les formes des sociabilités juvéniles populaires, et notamment la culture anti-école marquant la culture juvénile ouvrière, se trouvaient davantage définies par leur relation avec la culture de l'atelier (et son anticipation) [Willis 1978] qu'avec la culture scolaire, et pouvaient être envisagées comme systèmes symboliques autonomes, en dehors de tout rapport scolaire, tel n'est plus le cas aujourd'hui. Les sociabilités juvéniles des enfants de familles populaires et leurs formes radicalisées, comme l'adoption en l'école de la culture de provocation, constituent en effet, en lien avec la destructuration du groupe ouvrier [Beaud, Pialoux 1999], l'un des avatars des contradictions de la "démocratisation» scolaire.

\section{BIBLIOGRAPHIE}

BAUTIER E., RocheX J.Y. (1997), "Apprendre : des malentendus qui font la différence », in TERRAIL J.P. (dir.), La scolarisation de la France. Critique de l'état des lieux, La Dispute, Paris, p. 105-122.

BEAUD S. (2002), $80 \%$ au bac... et après? Les enfants de la démocratisation scolaire, La Découverte, Paris.

Beaud S., Pialoux M. (1999), Retour sur la condition ouvrière, Fayard, Paris.

Beaud S., Pialoux M. (2003), Violences urbaines, violence sociale, Fayard, Paris.

${ }^{13}$. Au point que, pour nombre de familles ouvrières, elle constitue la « seule voie de salut social pour leurs enfants » et que l'on a pu parler à leur égard d'une véritable « fuite en avant » dans la scolarisation prolongée [Beaud, Pialoux 1999] ou d'un mot d'ordre tramant le rapport des familles populaires à l'école : « hors l'école, point de salut ! » [Thin 1998]. 
BERnSteIn B. (1975), Langages et classes sociales, Minuit, Paris.

Bordet J. (1998), Les "jeunes de la cité », Presses Universitaires de France, Paris.

BOURDIEU P., PASSERON J.C. (1970), La reproduction. Éléments pour une théorie du système d'enseignement, Minuit, Paris.

BRocCOLICHI S. (1998), "Qui décroche? », in BLOCH M.C., GERDE B. (dir.), Les lycéens décrocheurs. De l'impasse aux chemins de traverse, Chronique Sociale, Lyon, p. 39-51.

BRunETEAUX P. (1994), «Les institutions et les paumés : les logiques de la surenchère », Critiques Sociales, $\mathrm{n}^{\circ}$ 5-6, p. 107-120.

Castel R., Haroche C. (2001), Propriété privée, propriété sociale, propriété de soi, Fayard, Paris.

Chamboredon J.C. (1971), «La délinquance juvénile, essai de construction d'objet», Revue Française de Sociologie, vol. XII, n 3, p. 335377.

Glasman D., Euvrard F. (dir.) (2004), La déscolarisation, La Dispute, Paris.

Grignon C., PASSERON J.C. (1989), Le savant et le populaire, Gallimard-Le Seuil-EHESS, Paris.

Kherroubi M, Millet M., Thin D. (2005), Classes-relais et familles. Accompagnement ou normalisation?, Collection «Études et Recherches », $n^{\circ} 8$, CNFE-PJJ Vaucresson, Paris.

LAGRANGE H. (2001), De l'affrontement à l'esquive. Violences, délinquances et usages de drogues, Syros, Paris.

LAHIRE B. (1993), Culture écrite et inégalités scolaires, Presses Universitaires de Lyon, Lyon.

Lepoutre D. (1994), "Le langage, l'école et la rue», Critiques Sociales, $\mathrm{n}^{\circ}$ 5-6, p. 5-12.

LePOUtRe D. (1997), Cour de banlieue. Codes, rites et langages, Odile Jacob, Paris.

MAUGER G. (2001), «Disqualification sociale, chômage, précarité et montée des illégalismes ", Regards Sociologiques, $\mathrm{n}^{\circ}$ 21, p. 79-86.

Millet M., ThiN D. (2004), « La 'déscolarisation' comme parcours de disqualification symbolique », in Glasman D., CEuvrard F. (dir.), La déscolarisation, La Dispute, Paris, p. 265-278. 
Millet M., Thin D. (2005), Ruptures scolaires. L'école à l'épreuve de la question sociale, Presses Universitaires de France, Paris.

PASSERON J.C. (1982), «L'inflation des diplômes. Remarques sur l'usage de quelques concepts analogiques en sociologie », Revue Française de Sociologie, $\mathrm{n}^{\circ}$ XXIII, p. 551-584.

THIN D. (1998), Quartier populaire. L'école et les familles, Presses Universitaires de Lyon, Lyon.

THIN D. (1999), "Désordre " scolaire dans les collèges de quartiers populaires, GRS-Lyon II, Lyon, ronéo.

THIN D. (2002), «L'autorité pédagogique en question. Le cas des collèges de quartiers populaires », Revue Française de Pédagogie, $\mathrm{n}^{\circ} 139$, p. 21-30.

VINCENT G. (1980), L'École primaire française, Presses Universitaires de Lyon, Lyon.

WiLlis P. (1978), "L'école des ouvriers », Actes de la Recherche en Sciences Sociales, ${ }^{\circ}$ 24, p. 50-61. 